\section{Effect of Screening for Methicillin- Resistant Staphylococcus aureus Carriage by Polymerase Chain Reaction on the Duration of Unnecessary Preemptive Contact Isolation}

\author{
Ilker Uçkay, MD; Hugo Sax, MD; Anne Iten, MD; \\ Véronique Camus, RN; Gesuele Renzi, RN; \\ Jacques Schrenzel, MD; Arnaud Perrier, MD; \\ Didier Pittet, MD, MS
}

A high prevalence of methicillin-resistant Staphylococcus aureus (MRSA) carriage at hospital readmission among previous MRSA carriers warrants screening and preemptive isolation precautions. The replacement of culture on chromogenic agar with rapid quantitative polymerase chain reaction for readmission screening reduces the number of unnecessary preemptive isolation-days by $54 \%$ (from 6.88 to 3.14 isolation-days) and related costs by $45 \%$ (from US $\$ 113.2$ to US\$62.1) for patients who test negative for MRSA.

Infect Control Hosp Epidemiol 2008; 29:1077-1079

Infections caused by methicillin-resistant Staphylococcus aureus (MRSA) are associated with adverse outcomes and increased hospital costs.' Guidelines and expert opinion recommend that, in addition to transmission precaution measures, MRSA-positive patients should be placed in single rooms or cohorted with similarly colonized patients. ${ }^{2}$ Previous MRSA carriage is the principal risk factor for carriage at hospital readmission and is responsible for a large proportion of the institutional reservoir of MRSA patients. ${ }^{3-5}$ Therefore, it is common practice to screen for previous carriers and to place them in preemptive isolation at hospital readmission while awaiting the results of screening. ${ }^{4}$

Because of logistical problems, the cost of isolation, patient discomfort, and the potential loss of care, it is desirable to shorten the latency period between screening and reporting of the results. In hospitals where there are insufficient rooms for the isolation or cohorting of MRSA-positive patients, a lower prevalence of presumed MRSA colonization reduces the need to house MRSA carriers with noncarriers in rooms with multiple beds, and thus reduces the risk of transmission. The use of polymerase chain reaction (PCR) for screening can shorten this latency period considerably, more so than the use of culture on chromogenic agar. In our study, we quantified the impact on logistics and costs of replacing the use of culture on chromogenic agar with the use of rapid multiplex quantitative PCR ( $\mathrm{qPCR})^{6,7}$ for the screening of previous MRSA carriers at hospital admission.

\section{METHODS}

Setting. The University of Geneva Hospitals, a 2,200-bed tertiary healthcare center, had 48,073 admissions in 2006.
Previous MRSA carriers are flagged by a computerized rapid alert system. ${ }^{4}$ Our study was conducted in the general internal medicine service, which has 246 acute care beds, 9,249 annual admissions, and an average length of stay of 9.4 days. According to an audit in December 2006, 25\% of MRSA-positive patients were isolated in single rooms, $24 \%$ were in rooms with noncarriers, and $51 \%$ were cohorted in rooms with either 2 or 6 beds.

Study procedures. During 2 separate 90-day periods, all patients admitted to the hospital who were previous MRSA carriers were included in our study, independent of their length of hospitalization. The intervention period (from September 23 to December 21, 2006) differed from the control period (from January 1 to March 31, 2006) in the microbiological method used to process screening samples. During the intervention period, use of culture on chromogenic agar (MRSA ID; bioMérieux), which was the microbiological method normally used for MRSA screening, was replaced by the use of qPCR. ${ }^{6}$ During both periods, samples were obtained on 2 consecutive days from the anterior nares and the groin using a cotton swab moistened with sterile saline solution.

The main outcome variable was the number of unnecessary preemptive isolation-days among previous MRSA carriers who tested negative on readmission. The average cost for 1 isolation-day, including the use of 10 gowns and 10 pairs of gloves and assuming a nursing severity score of 3 points, was estimated at a very conservative US $\$ 15$ for each patient. To account for the different proportions of MRSA-negative patients during both periods, we assessed the number of isolation-days and the other costs incurred during the intervention period both in absolute numbers and by simulating the use of a chromogenic culture method for the interventionperiod population.

All patients with previous MRSA carriage were placed in single rooms or cohorted, if logistically feasible; otherwise, barrier precautions were applied to patients sharing a room with noncarriers. Preemptive isolation was stopped on the day that both admission samples were reported to have negative results. Decolonization (ie, nasal mupirocin treatment twice daily for 5 days and whole-body washing with chlorhexidine soap for 7 days) was performed for all patients who had screening results positive for MRSA and was repeated if unsuccessful.

\section{RES ULT S}

Overall, 1,583 hospital admissions accounted for 16,396 patient-days during the control period, and 1,942 hospital admissions accounted for 20,060 patient-days during the intervention period. For patients with MRSA carriage, there were a total of 1,570 isolation-days during the control period 
TABLE. Data on Patients Screened for Methicillin-Resistant Staphylococcus aureus (MRSA) Carriage Using 2 Different Screening Tests for 2 Separate Periods

\begin{tabular}{|c|c|c|c|c|c|}
\hline Period, patient group & $\begin{array}{l}\text { No. of } \\
\text { patients }\end{array}$ & $\begin{array}{c}\text { No. of } \\
\text { isolation-days }\end{array}$ & $\begin{array}{l}\text { Screening } \\
\text { cost, US\$ }\end{array}$ & $\begin{array}{l}\text { Isolation } \\
\text { cost, US\$ }\end{array}$ & $\begin{array}{c}\text { Overall } \\
\text { cost, US\$ }\end{array}$ \\
\hline \multicolumn{6}{|l|}{ Control period ${ }^{a}$} \\
\hline MRSA positive & 82 & 930 & 820 & 13,950 & 14,770 \\
\hline MRSA negative & 73 & 502 & 730 & 7,530 & 8,260 \\
\hline Total & 155 & 1,432 & 1,550 & 21,480 & 23,030 \\
\hline \multicolumn{6}{|l|}{ Intervention period ${ }^{\mathbf{b}}$} \\
\hline MRSA positive & 63 & 1,132 & 945 & 16,980 & 17,925 \\
\hline MRSA negative & 113 & 355 & 1,695 & 5,325 & 7,020 \\
\hline Total & 176 & 1,487 & 2,640 & 22,305 & 24,945 \\
\hline \multicolumn{6}{|c|}{ Simulated intervention period ${ }^{c}$} \\
\hline MRSA positive & 63 & 1,132 & 630 & 16,980 & 17,610 \\
\hline MRSA negative & 113 & 777 & 1,130 & 11,655 & 19,399 \\
\hline Total & 176 & 1,909 & 1,760 & 28,635 & 30,395 \\
\hline
\end{tabular}

NOTE. Two consecutive screenings were performed for each patient. The average interval between admission and reporting of negative test results to the ward was 6.88 days (95\% confidence interval, 6.41-7.35; median, 7 isolation-days) during the control period and 3.14 days (95\% confidence interval, $2.75-3.37$; median, 3 isolation-days) during the intervention period $\left(P<.001\right.$; by 2 -tailed $\chi^{2}$ test).

a Screening (from January 1 to March 31,2006 ) by chromogenic agar culture, which was US\$10 for each patient.

b Screening (from September 23 to December 21, 2006) by in-house quantitative polymerase chain reaction (qPCR), which was estimated at a very conservative US\$15 for each patient. For the use of commercial PCR (at US\$70 per patient), the screening cost was estimated to be US\$4,410 for MRSA-positive patients and US\$7,910 for MRSA-negative patients, with an overall cost of US $\$ 34,625$.

c Simulation by use of chromogenic agar culture.

and a total of 1,986 isolation-days during the intervention period. Data on the study patients are shown in the Table for the control and intervention periods.

The average interval between admission and reporting of MRSA-negative results to the ward was 6.88 days (95\% confidence interval, 6.41-7.35; median, 7 isolation-days) during the control period and 3.14 days (95\% confidence interval, 2.75-3.37; median, 3 isolation-days) during the intervention period $\left(P<.001\right.$; by 2 -tailed $\chi^{2}$ test), corresponding to a $54.4 \%$ reduction in unnecessary isolation-days. Admission screening costs were US\$10 for culture and US $\$ 15$ for inhouse $\mathrm{qPCR}$ (US $\$ 5$ and US $\$ 7.50$ per test, respectively). The average cost per patient related to preemptive contact isolation of previous MRSA carriers who tested negative for MRSA at readmission was $45 \%$ lower during the intervention period (US\$62.10) than during the control period (US\$113.20) (Figure).

\section{I S C USSION}

So far, the impact of screening known MRSA carriers at hospital readmission by PCR has only been evaluated for intensive care units, where this type of PCR screening reduced the number of isolation-days ${ }^{7}$ and the incidence of MRSA transmission. ${ }^{8}$ Our study is the first, to our knowledge, to evaluate the impact of screening known MRSA carriers at hosptial readmission by $\mathrm{qPCR}$, instead of in vitro culture, in acute care medicine wards, and the result was a reduction of a median of 4 isolation-days per patient and a reduction in related costs for previous MRSA carriers who tested negative at readmission.

The duration of contact isolation was prolonged by the requirement that patients test negative for 2 separate samples

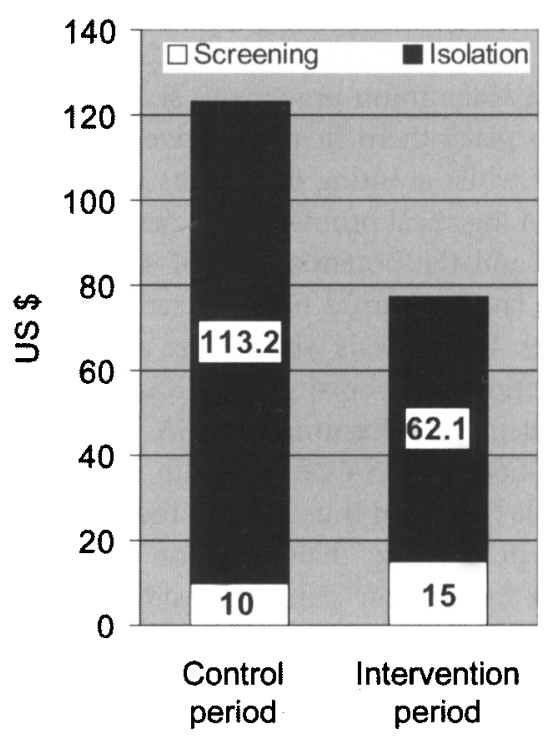

FIGURE. Total estimated average cost per patient for screening and isolation for the control period (with screening by culture) and the isolation period (with screening by in-house quantitative polymerase chain reaction). 
obtained on 2 consecutive days before contact isolation was stopped. By considering only the first screening by $\mathrm{qPCR}$ during the intervention period, we would have missed $7 \mathrm{pa}$ tients ( $11 \%$ of all MRSA-positive patients) but would have saved an additional 126 isolation-days, with a possible detrimental impact on patient safety.

The overall benefit of switching to a more rapid screening test depends on 3 key parameters: the number of patients at admission who are previous MRSA carriers; the proportion of these patients positive for MRSA; and the mean duration of contact isolation for MRSA-positive patients. The present real-life assessment demonstrates that these parameters may vary over time, even in the same hospital with the same policy for screening and contact isolation. Indeed, because of the higher number of study patients, in general, and the longer duration of contact isolation for MRSA-positive patients, in particular, the gross expenditure was slightly higher during the intervention period. To control for these confounders, we simulated the use of the chromogenic culture method for the intervention period population. In this simulation model, the use of qPCR resulted in an overall reduction in the number of isolation-days (422 [22.1\%] of 1,909 isolation-days were saved) and in total cost (US\$5,450 [17.9\%] of US $\$ 30,395$ were saved).

Our PCR cost estimates were based on use of an in-house PCR assay. However, most hospitals use commercial PCR assays, which may cost US $\$ 28-U S \$ 42$. Considering an "average" PCR at US $\$ 35$ per test, we found that the simulated costs would have risen by $13.9 \%$ if 2 PCR screenings were performed (Table) but would still have been reduced by $3.6 \%$ if only a single PCR test (with a single culture) was performed, which better corresponds to the reality in many hospitals.

The cost of isolation may vary according to the hospital setting. Our cost estimates are very conservative. For a neonatal intensive care unit, Karchmer et al. ${ }^{9}$ reported minimal costs of US $\$ 30$ per isolation-day for material (ie, gloves, gowns, and masks) and time.

Since the introduction of our in-house $\mathrm{qPCR}$, its sensitivity has been shown to be $96 \%$, its specificity $91 \%$, and its negative predictive value more than $99 \%$, compared with the standard culture technique (unpublished data). Similarly, widely used commercial tests showed negative predictive values greater than $97 \% .^{8,10}$

Because of the logistic and financial challenges for hospitals to control MRSA, it might be worth introducing PCR for MRSA screening, in addition to the promotion of hand hygiene, efficacious contact isolation, and better decolonization procedures. Because the introduction of PCR depends on several parameters, each institution should individually evaluate the possible benefits in advance. Currently, we are extending the use of qPCR to other sectors of our healthcare facility for the screening of previous MRSA carriers at hospital readmission.

\section{ACKNOWLEDGMENTS}

We are indebted to Rosemary Sudan for providing editorial assistance, to the nursing team of the general internal medicine service for their collaboration, to Dr. Stephan Harbarth for his expert opinion, and to the team of the Central Laboratory of Bacteriology of the University of Geneva Hospitals for the analyses.

Potential conflicts of interest. All authors report no conflicts of interest relevant to this article.

From the Infection Control Program (I.U., V.C., D.P.), the Service of General Internal Medicine (A.I., A.P.), and the Central Laboratory of Bacteriology (G.R., J.S.), University of Geneva Hospitals and Faculty of Medicine, Geneva, Switzerland.

Address reprint requests to Didier Pittet, MD, MS, Infection Control Program, University of Geneva Hospitals and Facuty of Medicine, 24 Rue Micheli-du-Crest 1211, Geneva 14, Switzerland (didier.pittet@hcuge.ch).

Received April 1, 2008; accepted June 17, 2008; electronically published October 9, 2008.

(C) 2008 by The Society for Healthcare Epidemiology of America. All rights reserved. 0899-823X/2008/2911-0013\$15.00. DOI: $10.1086 / 591452$

\section{REFERENCES}

1. Cosgrove SE, Qi Y, Kaye KS, et al. The impact of methicillin resistance in Staphylococcus aureus bacteremia on patient outcomes: mortality, length of stay, and hospital charges. Infect Control Hosp Epidemiol 2005; 26:166-174.

2. Siegel JD, Rhinehart E, Jackson M, Chiarello L; Healthcare Infection Control Practices Advisory Committee. Management of multidrug-resistant organisms in healthcare settings, 2006. Available at: http:// www.cdc.gov/ncidod/dhqp/pdf/ar/mdroguideline2006.pdf. Accessed January $10,2008$.

3. Harbarth S, Sax H, Fankhauser-Rodriguez C, Schrenzel J, Agostinho A, Pittet D. Control of a cluster of community-associated, methicillin-resistant Staphylococcus aureus in neonatology. J Hosp Infect 2006; 63:93100.

4. Pittet D, Safran E, Harbarth S, et al. Automatic alerts for methicillinresistant Staphylococcus aureus surveillance and control: role of a hospital information system. Infect Control Hosp Epidemiol 1996; 17:496-502.

5. Rubinovitch B, Pittet D. Screening for methicillin-resistant Staphylococcus aureus in the endemic hospital: what have we learned? I Hosp Infect 2001; 47:9-18.

6. François P, Pittet D, Bento $M$, et al. Rapid detection of methicillinresistant Staphylococcus aureus directly from sterile or nonsterile clinical samples by a new molecular assay. J Clin Microbiol 2003; 41:254-260.

7. Harbarth S, Masuet-Aumatell C, Schrenzel J, et al. Evaluation of rapid screening and pre-emptive contact isolation for detecting and controlling methicillin-resistant Staphylococcus aureus in critical care: an interventional cohort study. Crit Care 2006; 10:R25.

8. Cunningham R, Jenks P, Northwood J, et al. Effect on MRSA transmission of rapid PCR testing of patients admitted to critical care. J Hosp Infect 2007; 65:24-8.

9. Karchmer TB, Durbin LJ, Simonton BM, Farr BM. Cost-effectiveness of active surveillance cultures and contact/droplet precautions for control of methicillin-resistant Staphylococcus aureus. J Hosp Infect 2002; 51:126132.

10. Wagenvoort JH, van de Cruijs MF, Meuwissen CT, et al. Comparison of an enrichment broth-enhanced commercial PCR procedure versus bacteriological culture for separating non-colonized from suspected or colonized MRSA individuals. Eur J Clin Microbiol Infect Dis 2007; 26:155160. 